\title{
On the Spectrum of Internal Symmetries in the Algebraic Quantum Field Theory
}

\author{
A. Z. JADCZYK \\ Institute of Theoretical Physics \\ University of Wrocław
}

Received October 14, 1968

\begin{abstract}
It is shown that the point spectrum of internal symmetries is always symmetric. It is a group provided the intersection of all local subalgebras is trivial.
\end{abstract}

\section{Introduction}

The algebraic quantum field theory is based on the notion of algebras of observables. These are either abstract $C^{*}$-algebras [1] or von Neumann algebras of operators $[2,3]$. We shall be concerned with these latter (which is quite natural since we intend to deal with conserved symmetries). In the algebraic approach any possible physical symmetry of the quantum system is mathematically represented as an automorphism of the algebra of all observables. The symmetry is conserved, if the vacuum is invariant under the action of unitary operators implementing the associated automorphism. In [4-8] the conditions under which a given symmetry is conserved has been extensively studied in a connection with the Goldstone theorem. We shall be concerned with symmetries which are conserved. Space-time translations are such symmetries (by a definition!). These are geometric ones. Aside from them, there also occur symmetries which map each local subalgebra onto itself. They are called internal symmetries. The gauge-type transformations are of this kind.

It should be noticed that, from the purely mathematical point of view, the situation under our consideration is similar to that which occurs in statistical mechanics, when we deal with states of the thermal equilibrium of infinite systems. Consequently, a small modification of the methods used in [11] in studying the spectrum of space and time translations enables us to obtain similar results, in this case, for the spectrum of internal symmetries.

\section{General Assumptions and Notation}

For the sake of completeness we shall list once more the basic postulates of the algebraic quantum field theory. 
Let $\mathscr{L}(\mathscr{H})$ be an algebra of all bounded, linear operators acting on a Hilbert space $\mathscr{H}$. Let $\mathfrak{M}$ be a subset of $\mathscr{L}(\mathscr{H})$. The set $\mathfrak{M}^{\prime}$ of all $B$ in $\mathscr{L}(\mathscr{H})$ which commute with all $A$ in $\mathfrak{M}$ is then called a commutant of $\mathfrak{M}$. If $\mathfrak{M}=\mathfrak{W}^{\prime \prime}$ or equivalently if $\mathfrak{M}$ is a weakly closed $*$-subalgebra of $\mathscr{L}(\mathscr{H})$ containing the identity operator $I$, then $\mathfrak{M}$ is said to be a von Neumann algebra. For every $\mathfrak{M} \subset \mathscr{L}(\mathscr{H}), \mathfrak{W}^{\prime \prime}$ is the smallest von Neumann algebra containing the set $\mathfrak{M}$ - the von Neumann algebra generated by $\mathfrak{M}$.

The Postulates

1. To every bounded, open region $\mathcal{O}$ of space-time is associated a ron Neumann algebra $\mathscr{A}(\mathcal{O})$ in such a way that

a) if $\mathcal{O}_{1} \subset \mathcal{O}_{2}$ then $\mathscr{A}\left(\mathcal{O}_{1}\right) \subset \mathscr{A}\left(\mathcal{O}_{2}\right)$;

b) if $\left\{\mathcal{O}_{n}\right\}$ is an open covering of space-time, then the von Neumann algebra generated by $\cup \mathscr{A}\left(\mathcal{O}_{n}\right)$ and the von Neumann algebra generated by all $\mathscr{A}(\mathcal{O})$ coincide:

$$
\mathscr{A}=\left[\cup \mathscr{A}\left(\mathcal{O}_{n}\right)\right]^{\prime \prime}=[\cup \mathscr{A}(\mathcal{O})]^{\prime \prime}
$$

c) if $\mathcal{O}_{1}$ and $\mathcal{O}_{2}$ are totally space-like regions, then $\mathscr{A}\left(\mathcal{O}_{1}\right) \subset \mathscr{A}\left(\mathcal{O}_{2}\right)^{\prime}$.

2 . There exists a strongly continuous representation $x \rightarrow T(x)$ of the translation group of $R^{4}$, such that

a) $T(x) \mathscr{A}(\mathcal{O}) T(x)^{-1} \subset \mathscr{A}(\mathcal{O}+x)$ for all $\mathcal{O}$ and $x \in R^{4}$;

b) the support of a spectral measure $d E(p)$ uniquely defined by

$$
T(x)=\int e^{i p x} d E(p)
$$

is contained in a (closed) future light-cone.

3 . There exists the "vacuum state" $\Omega$, invariant under all $T(x)$ and cyclic for $\mathscr{A}$ (i.e. the set $\mathscr{A} \Omega$ is everywhere dense in $\mathscr{H}$ ).

Under these assumptions the (generalized) Reeh and Schlieder theorem is valid [9]:

Theorem. Let $\mathscr{H}_{0}$ be a subspace of $\mathscr{H}$, cyclic for $\mathscr{A}$ and such that $T(x) \mathscr{H}_{0} \subset \mathscr{H}_{0}$ for all $x$ in $R^{4}$. Then $\mathscr{H}_{0}$ is also cyclic for each $\mathscr{A}(\mathcal{O})$. In particular, for each $\mathcal{O}$ we have $\mathscr{H}=\overline{\mathscr{L}(\mathcal{O}) \Omega}$, so that by 1., c) $\Omega$ is cyclic and separating for $\mathscr{A}(\mathcal{O})$.

With the above assumptions we shall proceed to the considerations of internal symmetries. Therefore we shall assume, once for all that.

4. There is a unitary, strongly continuous representation $\tau \rightarrow U_{\tau}$ of the locally compact group $G$, such that

a) $U_{\tau} \mathscr{A}(\mathcal{O}) U_{\tau}^{-1}=\mathscr{A}(\mathcal{O})$ for all $\mathcal{O}$ and $\tau \in G$;

b) $U_{\tau} \Omega=\Omega$ for all $\tau \in G$.

It should be remarked at this point that we loose nothing by considering only continuous representation of $G$. Any group $G$ may be thought of as locally compact and any representation of $G$ may be thought of to be continuous. Namely, it is sufficient to equip $G$ in a discrete topology. 


\section{Properties of the Spectrum}

In what follows we shall study the spectrum of $U_{G}$. Our definition of the spectrum is a rather abstract one. Let $\chi$ be a character of $G$. By a character we mean a continuous homomorphism of $G$ into a group $\left\{e^{i \alpha}: 0 \leqq \alpha \leqq 2 \pi\right\}$. Let $F(\chi)$ be an orthogonal projection onto a (closed) subspace $\left\{\psi \in \mathscr{H}: U_{\tau} \psi=\langle\chi, \tau\rangle \psi\right\}$. The character $\chi$ is said to be in the point spectrum of $U_{G}$ if $F(\chi) \neq 0$. Denoting by $S\left(U_{G}\right)$ the point spectrum of $U_{G}$ we see that by definition, $S\left(U_{G}\right)$ is a subset of the group $\hat{G}$ of all continuous characters of $G$.

It $G$ is abelian, then $\hat{G}$ is locally compact and by the S.N.A.G. theorem $[10$, p. 377]:

$$
U_{\tau}=\int\langle\chi, \tau\rangle d F(\chi)
$$

$\chi \in S\left(U_{G}\right)$ if and only if $F(\{\chi\})=F(\chi) \neq 0$. In particular if $\left\{U_{\tau}\right\}$ is $n$-parameter, then every $\chi \in S\left(U_{G}\right)$ is determined by unique $q$ such that $\langle\chi, \tau\rangle=\exp (i q \tau)$ and our definition coincides with a usual one.

The following theorem gives a simple criterion, under which $U_{G}$ has only a point spectrum.

Theorem 3.1. Assume $G$ is compact, abelian group. Then

$$
\Sigma\left\{F(\chi): \chi \in S\left(U_{G}\right)\right\}=I .
$$

Proof. With $\phi, \psi$ in $\mathscr{H}$ denote by $y(\tau)$ the function $\tau \rightarrow\left(\phi, U_{\tau} \psi\right)$. Since $G$ is compact and $y$ is continuous, we have $y \in L^{2}(G)$ and by [10, p. 382], $y$ can be represented as a Fourier series $y(\tau)=\Sigma_{n} c_{n} \chi_{n}(\tau)$, $c_{n} \neq 0$. On the other hand, by the S.N.A.G. theorem we have

$$
y(\tau)=\int\langle\chi, \tau\rangle(\phi, d F(\chi) \psi) .
$$

Now, from the orthogonality of the system of all characters in $\hat{G}$ it follows that $c_{n}=\left(\phi, F\left(\chi_{n}\right) \psi\right)$, so that $\chi_{n} \in S\left(U_{G}\right)$. On the other hand, by $\Sigma_{n} c_{n}=y(e)=(\phi, \psi)$ we have

$$
(\phi, \psi)=\Sigma_{n}\left(\phi, F\left(\chi_{n}\right) \psi\right) .
$$

If $\psi$ is orthogonal to all $F(\chi) \phi$ with $\chi \in S\left(U_{G}\right)$, then, by the above $(\phi, \psi)=0$, so that the family $\left\{F(\chi): \chi \in S\left(U_{G}\right)\right\}$ of in pairs orthogonal projections spans the whole Hilbert space $\mathscr{H}$, what completes the proof.

Remark. It is clear that the assertion of the above theorem holds only if $G$ is abelian. Indeed, if the assertion holds, then the family $\left\{U_{r}\right\}$ of bounded operators has a complete set of simultaneous eigenvectors and thus all $U_{\tau}$ necessarily commute.

We now show that the point spectrum of $U_{G}$ is a subgroup of $\hat{G}$. This holds for an arbitrary, locally compact $G$ and follows from purely algebraic considerations. 
Theorem 3.2. The point spectrum of $U_{G}$ is symmetric i.e. $\chi \in S\left(U_{G}\right)$ implies $\chi^{-1} \in S\left(U_{G}\right)$. Moreover, if $\cap \mathscr{A}(\mathcal{O})=\{\lambda I\}$ then $S\left(U_{G}\right)$ is a subgroup of $G$ i.e. $\chi_{1}, \chi_{2} \in S\left(U_{G}\right)$ implies $\chi_{1} \chi_{2}^{-1} \in S\left(U_{G}\right)$.

Proof. The first assertion follows at once from [11, Corollary 4.2], since $\Omega$ is cyclic and separating for $\mathscr{A}(\mathcal{O})$. To prove the second let $\chi_{1}, \chi_{2} \in S\left(U_{G}\right)$ and $0 \neq \psi \in \dot{F}\left(\chi_{1}\right) \mathscr{H}$. Now, since $\psi$ is cyclic for an irreducible algebra $\cup \mathscr{A}(\mathcal{O})^{\prime}$ (we have $\left\{\cup \mathscr{A}(\mathcal{O})^{\prime}\right\}^{\prime \prime}=\{\cap \mathscr{A}(\mathcal{O})\}^{\prime}$ ) we can find $A$ in some $\mathscr{A}(\mathcal{O})^{\prime}$ such that $F\left(\chi_{2}\right) A \psi \neq 0$. But $\Omega$ is cyclic for $\mathscr{A}(\mathcal{O})$. Therefore we can choose $B$ in $\mathscr{A}(\mathcal{O})$ such that $\left(B \Omega, F\left(\chi_{2}\right) A \psi\right) \neq 0$. Let $\mathscr{M}$ be a Godement mean over $G$. Then, by [12, Theorem 1], for each $\chi$ in $\hat{G}$ we have

$$
(\phi, F(\chi) \psi)=\mathscr{M}\left\{\bar{\chi}(\hat{\tau})\left(\phi, U_{\hat{\tau}} \psi\right)\right\} .
$$

In particular $\left(B \Omega, F\left(\chi_{2}\right) A \psi\right)=\mathscr{M}\left\{\bar{\chi}_{2}(\hat{\tau})\left(B \Omega, U_{\hat{\tau}} A \psi\right)\right\}$. Now $A(\tau)$ $=U_{\tau} A U_{\tau}^{-1}$ is in $\mathscr{A}(\mathcal{O})^{\prime}$ for all $\tau$ and $U_{\tau} A \psi=\chi_{1}(\tau) U_{\tau} A U_{\tau}^{-1} \psi$ $=\chi_{1}(\tau) A(\tau) \psi$. Therefore

$$
\begin{aligned}
\left(B \Omega, F\left(\chi_{2}\right) A \psi\right) & =\mathscr{M}\left\{\bar{\chi}_{2}(\hat{\tau}) \chi_{1}(\hat{\tau})(B \Omega, A(\hat{\tau}) \psi)\right\} \\
& =\mathscr{M}\left\{\bar{\chi}_{2} \chi_{1}(\hat{\tau})\left(\Omega, A(\hat{\tau}) B^{*} \Omega\right)\right\} \\
& =\mathscr{M}\left\{\bar{\chi}_{2} \chi_{1}(\hat{\tau})\left(\Omega, A U_{\tau}^{-1} B^{*} \Omega\right)\right\} \\
& =\left(\Omega, A F\left(\chi_{1} \chi_{2}^{-1}\right) B^{*} \psi\right) .
\end{aligned}
$$

In this way we have $0 \neq\left(B \Omega, F\left(\chi_{2}\right) A \psi\right)=\left(\Omega, A F\left(\chi_{1} \chi_{2}^{-1}\right) B^{*} \psi\right)$ so that $F\left(\chi_{1} \chi_{2}^{-1}\right) \neq 0$. Q.E.D.

We have assumed in the last theorem that there is no non-trivial observable which can be measured in each $\mathscr{A}(\mathcal{O})$. This condition excludes the trivial case of abelian $\mathscr{A}$ and is clearly satisfied if $\Omega$ is a unique vector invariant under space-time translations. But this also holds for reducible $\mathscr{A}$ provided $\mathscr{A}(\mathcal{O})$ is a factor for at least one $\mathcal{O}$.

Now, let $G$ be abelian and $D \subset \hat{G}$ open, with $F(D) \neq 0$. Let $\mathcal{O}$ be arbitrary. By the cyclicity of $\Omega$ for $\mathscr{A}(\mathcal{O})$ one can find $A \in \mathscr{A}(\mathcal{O})$ such that $F(D) A \Omega \neq 0$. The same must hold for some compact set $D_{0} \subset D$. Let $f(\tau)$ be a function in $L^{1}(G)$ such that $\hat{f}(\chi)=\int \chi(\tau) f(\tau) d \mu(\tau)$ is in $L^{1}(G)$, positive, vanishing outside $D$ and equal one on $D_{0}$. Such function exists by $\left[10\right.$, p. 375]. Let $A_{f}=\int A(\tau) f(\tau) d \mu(\tau)$. Then $A_{f} \in \mathscr{A}(\mathcal{O})$ and $A_{f} \Omega=\int \hat{f}(\chi) d F(\chi) A \Omega={ }_{D} \int \hat{f}(\chi) d F(\chi) A \Omega$ so that $A_{f} \Omega \neq 0$ and $F(D) A_{f} \Omega=A_{f} \Omega$. On the other hand $A_{f}^{*} \Omega=\int f\left(\chi^{-1}\right) d F(\chi) A^{*} \Omega$. Therefore $F\left(D^{-1}\right) A_{f}^{*} \Omega=A_{f}^{*} \Omega$ and $A_{f}^{*} \Omega \neq 0$ since $\Omega$ is separating for $\mathscr{A}(\mathcal{O})$. So we obtain

Corollary 3.3. If $G$ is abelian, then supp $d F$ is a symmetric subset of $\hat{G}$ i.e. $\chi \in \operatorname{supp} d F$ implies $\chi^{-1} \in \operatorname{supp} d F$.

If $\mathscr{A}$ is irreducible, if the symmetry commutes with translations and if some continuity requirements are satisfied then, one can prove that supp $d F$ is a subgroup of $\hat{G}$. But it is quite possible that this also holds under considerably weaker assumptions. 


\section{The Case of $\boldsymbol{G}$ Compact}

Throughout this section $G$ will be compact, abelian. Denoting by $N$ the kernel of the representation $G \rightarrow U_{G}$ it is plain that $G / N$ is compact, abelian and the mapping $\tau \rightarrow U_{\tau}$ is a superposition of the canonical homomorphism $G \rightarrow G / N$ and an isomorphism $G / N \rightarrow U_{G}$. Since we are interested in properties of operators in a Hilbert space rather, then in representation theory, we shall restrict ourselves to the case of $N=\{e\}$. In other words we shall confine ourselves to the study of the quotient group $G_{0}=G / N$. With this in mind we have the following.

Theorem 4.1. The point spectrum of $U_{G}$ is identic with a character group $\hat{G}$ of $G$.

Proof. Since $\hat{G}$ is discrete, it follows that $\hat{S} \stackrel{\text { dt }}{=} S\left(U_{G}\right)$ is a closed subgroup of $\hat{G}$. Let $A(G, \hat{S})$ be the annihilator $\hat{S}$ in $G$ i.e. the subset of $G$ consisting of all $\tau$ such that $\chi(\tau)=1$ for all $\chi$ in $\hat{S}$ (see e.g. [13], p. 365 and further). Now, by [13, Theorem 24.10] we have $\widehat{S}=A(\hat{G}, A(G, \hat{S}))$. On the other hand it is trivial that $A(\hat{G},\{e\})=\hat{G}$. Thus it is sufficient to show that $A(G, \widehat{S})=\{e\}$. To prove this take an arbitrary $\tau_{0}$ in $A(G, \widehat{S})$. Then for each $\chi$ in $\hat{S}$ we have $\chi\left(\tau_{0}\right)=1$, or equivalently $U_{\tau_{0}} F(\chi)=F(\chi)$ for all $\chi$ in $\hat{S}$. But since $\{\Sigma F(\chi): \chi \in \hat{S}\}=I$, it follows that $U_{\tau_{0}}=I$ or $\tau_{0}=e$. Q.E.D.

It can be seen from the above that our conjecture is that every internal symmetry describes some kind of a charge, or better, a mixture of charges. If we want to deal with one pure charge only, then it is natural to expect that the spectrum is a cyclic infinite group i.e. that there is an "elementary" charge $\chi_{0}$, and all other are obtaining by taking succesive powers of $\chi_{0}$. Such a situation arises when $G=T$. (We denote by $T$ the group $\left\{e^{i \alpha}: 0 \leqq \alpha \leqq 2 \pi\right\}$.) Then $\hat{G}=Z$ where $Z$ denotes the (discrete) group of all integers, and the charge can take values: $0, \pm 1$, \pm 2 , etc. It is obvious that the converse is also true. Actually, if the spectrum of $U_{G}$ is a cyclic infinite group, then it is isomorphic with $Z$ and by the Duality theorem $(\widehat{\hat{G}} \cong G) G$ is isomorphic with $T$. Thus we have

Corollary 4.2. The symmetry group $G$ describes one pure charge only (i.e. the spectrum of $U_{G}$ is an infinite, cyclic group) if and only if $G$ is isomorphic with $T$.

If $G$ is connected then $\hat{G}$ (if nontrivial) is infinite and we deal with a charge which necessarily takes an infinite number of values. Thus if we want to describe a charge with a finite number of values then $G$ has to be disconnected.

If we look to the internal symmetries from the exhibited standpoint then the following question arises: it was argued in [1], that the field theory can be described using the zero-charge sector only. In what sense 
is that true if the charge is defined as above, by internal symmetry? We will give a partial answer to this and related questions in a subsequent paper.

Acknowledgements. The author is greatly indebted to Professor J. T. ŁopusZAŃSKI for his interest, for fruithful discussions and for reading the manuscript.

\section{References}

1. HAAG, R., and D. Kastler: J. Math. Phys. 5, 848 (1964).

2. 一, and B. Schroer: J. Math. Phys. 3, 248 (1962).

3. ARAKI, H.: Lecture Notes. Zürich ETH (1961).

4. Kastler, D., D. W. Robinson, and J. A. Swieca: Preprint.

5. Robrisson, D. W.: CERN preprint 66/1205/5-TH. 708.

6. ReEH, H.: Nuovo Cimento X, 51 A, 638 (1967).

7. - Preprint of the lecture presented at the $\mathrm{V}^{\mathrm{th}}$ Winter School of Theoretical Physics. Karpacz 1968.

8. Lopuszański, J.: On the Goldstone theorem. Preprint (1967).

9. Uhlmann, A.: Preprint Nr. 3/71. Wrocław 1964.

10. NAIMARK, M. A.: Normed rings. Moskva: Gostehizdat 1956.

11. JADCZYK, A. Z.: On the KMS-algebra. Preprint (1968).

12. Doplicher, S., and D. KastLer: IHES Preprint X 011 (1967).

13. HEwitT, E., and K. A. Ross: Abstract harmonic analysis. Berlin-GöttingenHeidelberg: Springer 1963.

Dr. A. Z. JADCZYK

Institute of Theoretical Physics

University of Wrockaw

ul. Cybulskiego 36

Wrocław, Poland 